\title{
NORTH-HOLLAND
}

\section{Adaptive Control of Uncertain Coupled Mechanical Systems with Application to Base-Isolated Structures}

\section{J. Rodellar}

Department of Applied Mathematics III

Technical University of Catalonia

Gran Capitán $s / n$

08034-Barcelona, Spain

E. P. Ryan

School of Mathematical Sciences

University of Bath

Claverton Down

Bath BA2 7AY, England

and
A. H. Barbat
Department of Structural Mechanics
Technical University of Catalonia
Gran Capitán $s / n$
08034-Barcelona, Spain

Transmitted by F. E. Udwadia

\section{ABSTRACT}

A problem of feedback stabilization is addressed for a class of uncertain nonlinear mechanical systems with $n$ degrees of freedom and $n_{c}<n$ control inputs. Each system of the class has the structure of two coupled subsystems with $n_{c}$ and $n_{r}$ degrees of freedom, respectively, a prototype being an uncertain base isolated building structure with $n$ degrees of freedom actively controlled via actuators applying forces to specific degrees of freedom of the base movement, $n_{c}<n$ in number. A nonlinear adaptive feedback strategy is described, which, under appropriate assumptions on the system uncertainties, guarantees a form of practical stability of the zero state. Numerical simulations are also presented to illustrate the application of the control strategy to a base isolated building. 


\section{INTRODUCTION}

The problem of actively controlling structures has been extensively studied in the last two decades. Whilst in many cases controllers have been designed under the assumption of having a perfectly known structural model, there has also been considerable interest in questions of robustness. Among the approaches proposed in the literature for the control of uncertain systems, there is one in which the systems are described by differential equations, the uncertainties are modeled deterministically, and Lyapunov techniques are used constructively to design feedback controllers to render the system "practically stable" (see [1] for an overview and extensive bibliography). This approach has been adopted for active control of structures in previous work [2-4].

Although much of the literature has dealt with fixed-parameter controllers, the design of adaptive control laws has also been considered $[5,6]$. In this context, the word adaptive means that the control law is parameterized by a variable gain whose value is autotuned according to some appropriately designed law. The present paper essentially falls into this category and focuses on the construction of adaptive convrollers for a class of uncertain nonlinear coupled inechanical system that can be decomposed into two subsystems with feedback control acting on one of them. the control law is developed in the context of this general class of systems, the problem of actively controlling a base-isolated building structure is our

\section{THE CLASS OF SYSTEMS}

We consider a class of uncertain mechanical systems $\Sigma$ with $n$ degrees of freedom and $n_{c}<n$ control inputs. Each system of the class has the structure of two coupled subsystems, $\Sigma_{c}$ and $\Sigma_{r}$, with $n_{c}$ and $n_{r}$ degrees of freedom, respectively, $n=n_{c}+n_{r}$, and described by equations of motion of the following form:

$$
\begin{aligned}
\Sigma_{r}: & M_{r}\left(q_{r}(t)\right) \ddot{q}_{r}(t)+g_{r}\left(q_{r}(t), \dot{q}_{r}(t)\right)=h\left(q_{c}(t), \dot{q}_{c}(t)\right), \\
& \left(q_{r}\left(t_{0}\right), \dot{q}_{r}\left(t_{0}\right)\right)=\left(q_{r}^{0}, v_{c}^{0}\right) \\
\Sigma_{c}: & M_{c}\left(q_{c}(t)\right) \ddot{q}_{c}(t)+g_{c}\left(t, q_{r}(t), \dot{q}_{r}(t), q_{c}(t), \dot{q}_{c}(t)\right)=u(t), \\
& \left(q_{c}\left(t_{0}\right), \dot{q}_{c}\left(t_{0}\right)\right)=\left(q_{c}^{0}, v_{c}^{0}\right) .
\end{aligned}
$$

Here, $q_{r}(t) \in \mathbb{R}^{n_{r}}, q_{c}(t) \in \mathbb{R}^{n_{c}}$ are vectors of generalized coordinates and $u(t) \in \mathbb{R}^{n_{c}}$ is the vector of control forces; the matrix-valued functions $M_{r}$ 
and $M_{c}$ represent inertias, and the (nonlinear) functions $g_{r}, h, g_{c}$ model damping, stiffness, coupling, and Coriolis effects, as well as extraneous inputs and disturbances acting on the overall system.

Assumptions 1 to 6 below complete the description of the system class $\Sigma$.

Assumption A1. The function $M_{r}$ is continuous with uniformly bounded inverse, that is, for some (unknown) positive scalar $\tilde{m},\left\|M_{r}^{-1}\left(q_{r}\right)\right\|$ $\leq \tilde{m}$ for all $q_{r} \in \mathbb{R}^{n_{r}}$.

Assumption A2. The function $M_{c}$ is continuous and such that, for some (unknown) positive scalars $\bar{m}, \underline{m}$ and known continuous function $\mu$, the following hold for all $q_{c} \in \mathbb{R}^{n_{c}}:(\mathrm{i})\left\|M_{c}^{-1}\left(q_{c}\right)\right\| \leq \bar{m} \mu\left(q_{c}\right)$, and (ii) $M_{c}^{-1}\left(q_{c}\right)$ $\geq \underline{m I}$ (in the sense that $\left\langle v, M_{c}^{-1}\left(q_{c}\right) v\right\rangle \geq \underline{m}\|v\|^{2}$ for all $v \in \leq p r^{n_{c}}$ ).

\section{Assumption A3. The function $g_{r}$ is continuous.}

\section{Assumption A4. The function $h$ is continuous, with $h(0,0)=0$.}

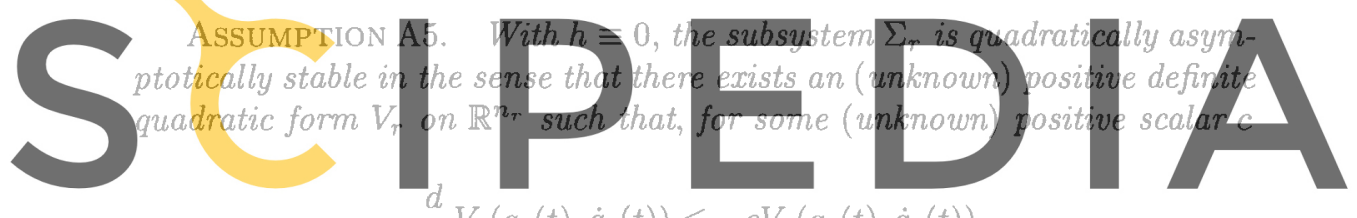

Register for free at https//wudt. scipedia.com to download the version without the watermark

for almost all t on every solution $\left(g_{r}, \dot{a}_{r}\right)(f)$.

Assumption A6. The function $g_{c}$ is of Carathéodory class and such that, for some known continuous function $\gamma$, the following holds for some (unknown) scalar $\alpha$ :

$$
\left\|g_{c}\left(t, q_{r}, v_{r}, q_{c}, v_{c}\right)\right\| \leq \alpha \gamma\left(q_{r}, v_{r}, q_{c}, v_{c}\right)
$$

for almost all $t \in \mathbb{R}$ and all $\left(q_{r}, v_{r}, q_{c}, v_{c}\right) \in \mathbb{R}^{2 n}$.

Thus, the only a priori system information available to the controller is the pair of continuous functions $\gamma$ and $\mu$ : in particular, we stress that the uncertainty bounding parameters $\tilde{m}, \underline{m}, \bar{m}$, and $\alpha$ are unknown.

The question to be addressed can be posed as follows: Does there exist an adaptive feedback strategy, parameterized by $\lambda>0$, which, for every system (unknown to the controller) of class $\Sigma$, every solution of the feedback-controlled initial-value problem (1) is asymptotic to a ball centred 
at zero in $\mathbb{R}^{n}$ of radius $\rho(\lambda)$, where $\rho(\lambda) \rightarrow 0$ as $\lambda \rightarrow 0$ ? In Section 3 , we answer this question affirmatively by explicit construction of one such feedback strategy.

\subsection{Example: Active control of base-isolated structures}

In the field of civil engineering structures there exists a great interest in reducing the structural response produced by seismic ground motions. In recent years, as one of the possibilities to achieve this objective, hybrid control systems have been proposed which combine base isolators with active control systems.

Base isolators attempt to uncouple the structure from the seismic ground motion by means of replaceable devices, placed between the building and the foundation, capable of absorbing part of the energy induced by earthquakes [7]. The base isolation component can reduce by itself both the interstory drift and the absolute accelerations of the structure. Thus the structure tends to behave like a rigid body, the price paid being a significant displacement of the base. Another drawback of such systems is the

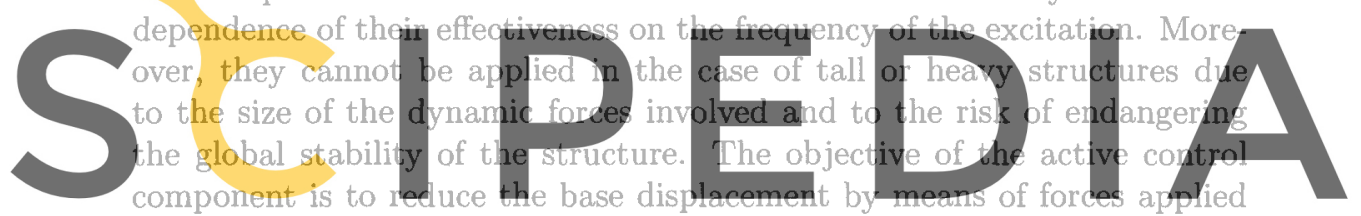

on the base. From a practical point of view, this hybrid scheme is appeal-

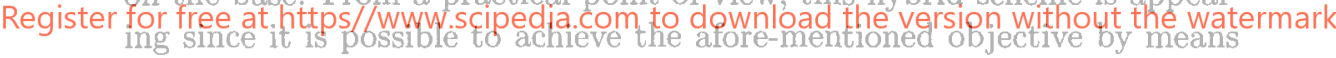
of a single force which, moreover, does not exceed some acceptable limits due to the high flexibility of the base isolators. Moreover, the active control action essentially does not depend on the frequency content of the dynamic excitation. From a theoretical point of view, the development of a control law to calculate the active force involves difficulties associated with the nonlinear behavior of the base isolators and to the uncertainties in the models describing the structure-base isolator system and in the seismic excitation.

A robust control law for linear systems has been proposed in a previous work [2]. Also for linear systems, the application of predictive control has been considered [8] as well as a form of bang-bang control [9]. The nonlinearity of the isolators has been considered in [10], assuming no uncertainties in the structure-base model. Some experimental works with small-scale hybrid systems have been recently reported $[11,12]$.

The hybrid control problem we are dealing with can be cast within the framework of the class of systems defined by (1). In the remaining of this section the equations of motion governing this problem will be presented. 
The dynamic behavior of the structure with the hybrid control system can be described by means of a model composed of two coupled systems: $\Sigma_{r}$ (the building) and $\Sigma_{c}$ (the base). It is assumed that the structure has a linear behavior due to the effect of the base isolation. The behavior of the isolator may be nonlinear. The vector $q_{r}$ represents the horizontal displacements of the $n$ degrees of freedom respect to an inertial frame, while the displacement of the structural base is described by a single degree of freedom with horizontal displacement $q_{c}$ relative to the afore-mentioned frame. The dynamic excitation is produced by a horizontal seismic ground motion, characterized by a displacement $d(t)$ and its velocity $v(t)$. A single horizontal control force $u(t)$ acts upon the structural base. Thus, the equations of motion are

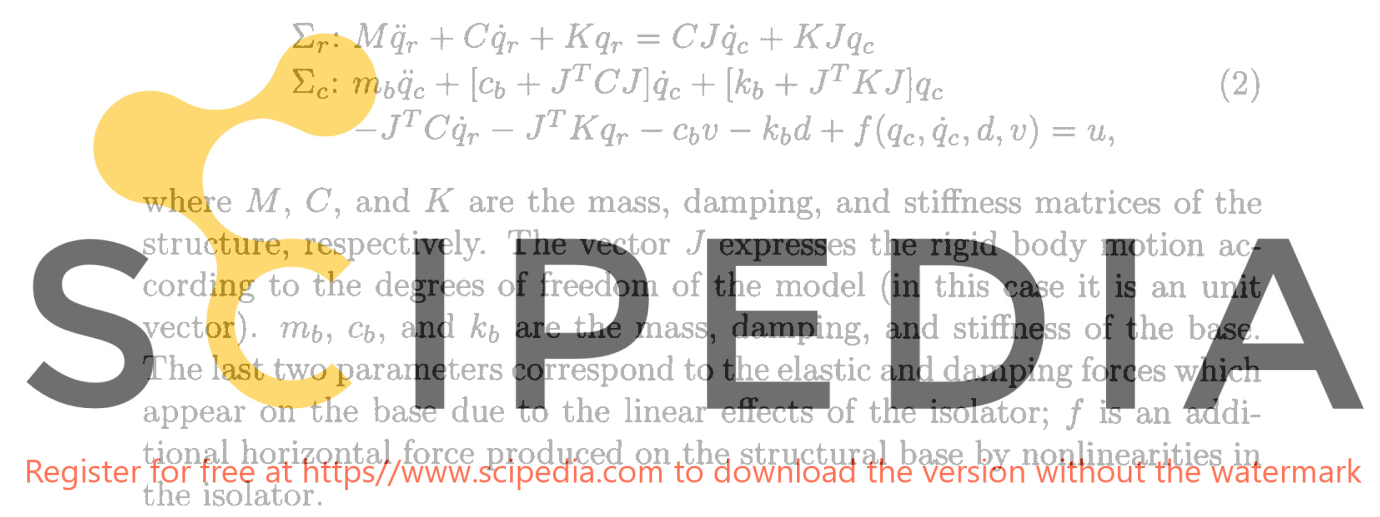

Assumptions $A 1-A 5$ hold as $M$ is invertible, $m_{b}>0$ and $C$ and $K$ are positive definite. Assumption A6 holds under the following conditions:

$$
\begin{aligned}
\left\|c_{b} v(t)+k_{b} d(t)\right\| & \leq \nu \\
\left\|f\left(q_{c}, \dot{q}_{c}, d, v\right)\right\| & \leq \alpha^{\prime} \gamma^{\prime}\left(q_{c}, \dot{q}_{c}\right)
\end{aligned}
$$

for almost all $t$ and all $\left(q_{c}, \dot{q}_{c}\right) \in \mathbb{R}^{2}, \nu$ and $\alpha^{\prime}$ being unknown scalars and $\gamma^{\prime}$ a known continuous function.

\section{THE ADAPTIVE STRATEGY}

Throughout this section, we assume $\lambda>0$. We first introduce some notation. Let $d_{\lambda}$ denote the function defined (on $\mathbb{R}^{n_{c}}, \mathbb{R}^{n_{r}}, \mathbb{R}^{2 n_{\mathrm{c}}}$, or $\mathbb{R}^{2 n_{r}}$ as context dictates) by

$$
d_{\lambda}: v \mapsto \begin{cases}\|v\|-\lambda, & \|v\| \geq \lambda \\ 0, & \|v\|<\lambda\end{cases}
$$


Let $s_{\lambda}$ denote the function defined on $\mathbb{R}^{n_{c}}$ by

$$
s_{\lambda}: v \mapsto \begin{cases}\|v\|^{-1} v, & d_{\lambda}(v)>0 \\ \lambda^{-1} v, & d_{\lambda}(v)=0\end{cases}
$$

The proposed adaptive strategy, parameterized by $\lambda>0$, is given by

$$
\begin{aligned}
u(t) & =-k(t) U_{\lambda}\left(q_{r}(t), \dot{q}_{r}(t), q_{c}(t), p_{c}(t)\right) \\
p_{c}(t) & =\dot{q}_{c}(t)+\eta q_{c}(t) \\
\dot{k}(t) & =K_{\lambda}\left(q_{r}(t), \dot{q}_{r}(t), q_{c}(t), p_{c}(t)\right) \\
k\left(t_{0}\right) & =k^{0}
\end{aligned}
$$

where $\eta>0$ (a design parameter) is open to choice, and the functions $U_{\lambda}$ and $K_{\lambda}$ are defined as follows:

\section{$U_{\lambda}:\left(q_{r}, v_{r}, q_{c}, p_{c}\right) \mapsto p_{c}+\gamma_{\mu}\left(q_{r}, v_{r}, q_{c}, p_{c}\right) s_{\lambda}\left(p_{c}\right)$ $K_{\lambda}:\left(q_{r}, v_{r}, p_{c}\right) \mapsto d_{\lambda}\left(p_{c}\right)\left[\left\|p_{c}\right\|+\gamma_{\mu}\left(q_{r}, v_{r}, q_{c}, p_{c}\right)\right]$ $\gamma_{\mu}:\left(q_{r}, v_{r}, q_{c}, p_{c}\right) \mapsto \mu\left(q_{c}\right) \gamma\left(q_{r}, v_{r}, q_{c}, p_{c}-\eta q_{c}\right)$.}

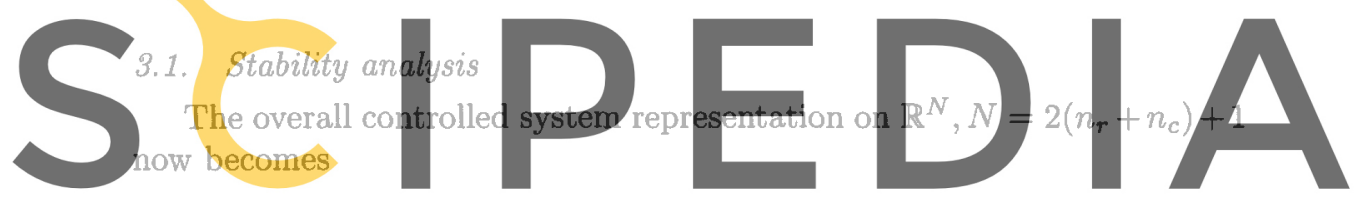

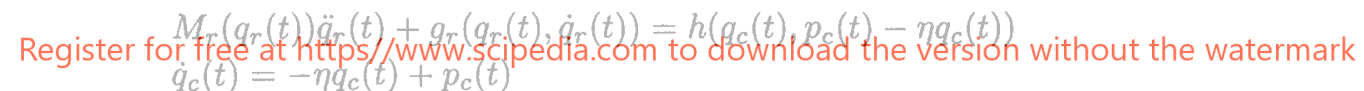

$$
\begin{aligned}
& \dot{p}_{c}(t)=P_{\lambda}\left(t, q_{r}(t), \dot{q}_{r}(t), q_{c}(t), p_{c}(t), k(t)\right) \\
& \dot{k}(t)=K_{\lambda}\left(q_{r}(t) \dot{q}_{r}(t), q_{c}(t), p_{c}(t)\right) \\
& \left(q_{r}\left(t_{0}\right), \dot{q}_{r}\left(t_{0}\right), q_{c}\left(t_{0}\right), p_{c}\left(t_{0}\right), k_{c}\left(t_{0}\right)\right)=\left(q_{r}^{0}, v_{r}^{0}, q_{c}^{0}, p_{c}^{0}, k^{0}\right)=: x^{0} \in \mathbb{R}^{N},
\end{aligned}
$$

where the function $P_{\lambda}$ is given by

$$
\begin{aligned}
& P_{\lambda}\left(t, q_{r}, v_{r}, q_{c}, p_{c}, k\right):=\eta p_{c}-\eta^{2} q_{c}-M_{c}^{-1}\left(q_{c}\right) \\
& \quad \times\left[g_{c}\left(t, q_{r}, v_{r}, q_{c}, p_{c}-\eta q_{c}\right)+k U_{\lambda}\left(q_{r}, v_{r}, q_{c}, p_{c}\right)\right] .
\end{aligned}
$$

Equivalently, writing $x(t)=\left(q_{r}(t), \dot{q}_{r}(t), q_{c}(t), p_{c}(t), k(t)\right)$,

$$
\dot{x}(t)=F_{\lambda}(t, x(t)), \quad x\left(t_{0}\right)=x^{0},
$$

where

$$
\begin{aligned}
F_{\lambda}: x=\left(q_{r}, v_{r}, q_{c}, p_{c}, k\right) \mapsto & \left(v_{r}, M_{r}^{-1}\left[h\left(q_{c}, p_{c}-\eta q_{c}\right)-g_{r}\left(q_{r}, v_{r}\right)\right]\right. \\
& \left.-\eta q_{c}+p_{c}, P_{\lambda}(t, x), K_{\lambda}(x)\right)
\end{aligned}
$$


This system satisfies the classical Carathéodory conditions and so, for every $\left(t_{0}, x^{0}\right) \in \mathbb{R} \times \mathbb{R}^{N}$, the above initial-value problem has a solution and every solution can be extended into a maximal solution.

On $[0, \infty)$, define

$$
\hat{h}: \lambda \mapsto \sup \left\{\left\|h\left(q_{c}, p_{c}-\eta q_{c}\right)\right\| \mid d_{\lambda}\left(q_{c}\right)=0=d_{\lambda}\left(p_{c}\right)\right\},
$$

which, by virtue of Assumption A4, is continuous with $\hat{h}(\lambda) \rightarrow 0$ as $\lambda \rightarrow 0$.

Theorem 1. Let $\lambda>0$ and $\left(t_{0}, x^{0}\right) \in \mathbb{R} \times \mathbb{R}^{N}$. For every maximal solution $x(\cdot)=\left(q_{r}, \dot{q}_{r}, q_{c}, p_{c}, k\right)(\cdot):\left[t_{0}, \omega\right) \rightarrow \mathbb{R}^{N}$ of the initial-value problem (7) (equivalently (8)),

(i) $\omega=\infty$;

(ii) $\lim _{t \rightarrow \infty} k(t)$ exists and is finite;

(iii) $d_{\lambda \eta-1}\left(q_{c}(t)\right)$ and $d_{\lambda}\left(p_{c}(t)\right) \rightarrow 0$ as $t \rightarrow \infty$;

(iv) for some positive scalar $c, d_{c \hat{h}(\lambda)}\left(q_{r}(t), \dot{q}_{r}(t)\right) \rightarrow 0$ as $t \rightarrow \infty$.
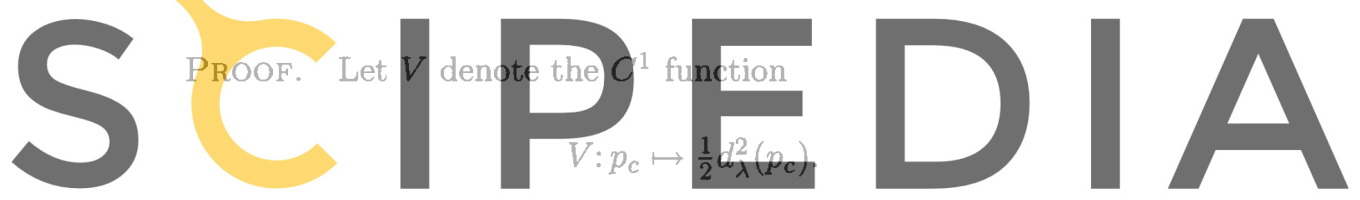

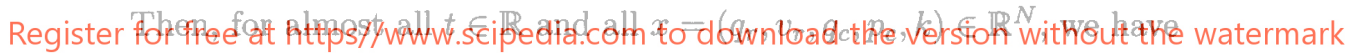

$$
\begin{gathered}
\left\langle\nabla V\left(p_{c}\right), P_{\lambda}(t, x)\right\rangle \leq a_{\lambda}\left(p_{c}\right)\left[\eta^{2}\left\|q_{c}\right\|+(\alpha \bar{m}-\underline{m} k) \gamma_{\mu}\left(q_{r}, v_{r}, q_{c}, p_{c}\right)\right. \\
\left.+(\eta-\underline{m} k)\left\|p_{c}\right\|\right] .
\end{gathered}
$$

Defining $k^{*}:=\underline{m}^{-1}(\eta+\alpha \bar{m})$, it follows that

$$
\frac{d}{d t} V\left(p_{c}(t)\right) \leq-\underline{m}\left(k(t)-k^{*}\right) \dot{k}(t)+\eta^{2} d_{\lambda}\left(p_{c}(t)\right)\left\|q_{c}(t)\right\|
$$

for almost all $t \in\left[t_{0}, \omega\right)$. Integration now yields, with $c_{0}:=V\left(p_{c}^{0}\right)+$ $\frac{1}{2} \underline{m}\left(k^{0}-k^{*}\right)^{2}$,

$$
0 \leq V\left(p_{c}(t)\right) \leq c_{0}-\frac{1}{2} \underline{m}\left(k(t)-k^{*}\right)^{2}+\eta^{2} \int_{t_{0}}^{t} d_{\lambda}\left(p_{c}(s)\right)\left\|q_{c}(s)\right\| d s,
$$

which is valid for all $t \in\left[t_{0}, \omega\right)$.

We briefly digress to prove a technicality. 
Proposition. For some positive scalar $c_{1}$,

$$
\int_{t_{0}}^{t} d_{\lambda}\left(p_{c}(s)\right)\left\|q_{c}(s)\right\| d s \leq c_{1} \int_{t_{0}}^{t}\left[d_{\lambda}\left(p_{c}(s)\right)+d_{\lambda}^{2}\left(p_{c}(s)\right)\right] d s
$$

for all $t \in\left[t_{0}, \omega\right)$.

Proof. First observe that

$$
\begin{aligned}
\left\|q_{c}(s)\right\| & \leq\left\|q_{c}^{0}\right\|+\int_{t_{0}}^{s} e^{-\eta(s-\sigma)}\left\|p_{c}(\sigma)\right\| d \sigma \\
& \leq\left\|q_{c}^{0}\right\|+\int_{t_{0}}^{s} e^{-\eta(s-\sigma)}\left[d_{\lambda}\left(p_{c}(\sigma)+\lambda\right] d \sigma\right. \\
& \leq\left\|q_{c}^{0}\right\|+\lambda+\int_{t_{0}}^{s} e^{-\eta(s-\sigma)} d_{\lambda}\left(p_{c}(\sigma)\right) d \sigma
\end{aligned}
$$

Therefore,

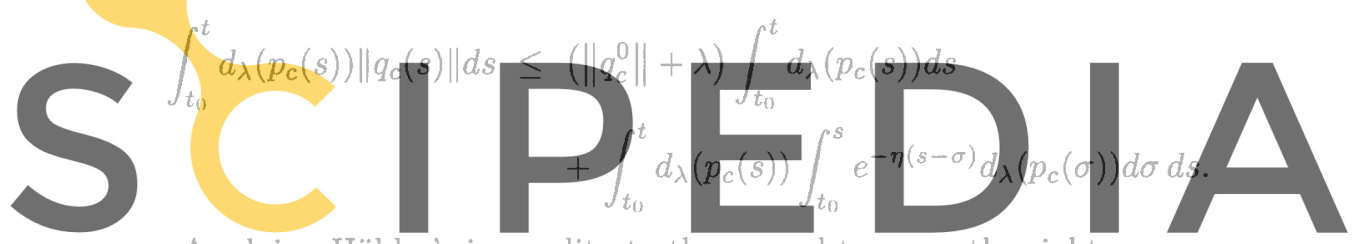

Applying Hölder's inequality to the second term on the right,

Register for free at https//www.scipedia.com to download the version without the watermark

$$
\begin{aligned}
\int_{t_{0}}^{t} d_{\lambda}\left(p_{c}(s)\right) \int_{t_{0}}^{s} e^{-\eta(s-\sigma)} d \lambda\left(p_{c}(\sigma)\right) d \sigma d s \leq\left(\int_{t_{0}}^{t} d_{\lambda}^{2}\left(p_{c}(s)\right) d s\right) & \\
& \times\left(\int_{t_{0}}^{t} e^{-2 \eta s}\left(\int_{t_{0}}^{t} e^{\eta \sigma} d_{\lambda}\left(p_{c}(\sigma)\right) d \sigma\right)^{2} d s\right)^{1 / 2} .
\end{aligned}
$$

Integrating by parts in the last term on the right

$$
\begin{aligned}
\int_{t_{0}}^{t} e^{-2 \eta s}\left(\int_{t_{0}}^{t} e^{\eta \sigma} d_{\lambda}\left(p_{c}(\sigma)\right) d \sigma\right)^{2} d s \leq & \int_{t_{0}}^{t} d_{\lambda}\left(p_{c}(s)\right) \\
& \times \int_{t_{0}}^{s} e^{-\eta(s-\sigma)} d_{\lambda}\left(p_{c}(\sigma)\right) d \sigma d s .
\end{aligned}
$$

We may now conclude that

$$
\int_{t_{0}}^{t} d_{\lambda}\left(p_{c}(s)\right) \int_{t_{0}}^{s} e^{-\eta(s-\sigma)} d_{\lambda}\left(p_{c}(\sigma)\right) d \sigma d s \leq \int_{t_{0}}^{t} d_{\lambda}^{2}\left(p_{c}(s)\right) d s
$$

whence the claim. 
Returning to the proof of the theorem, we now have

$$
0 \leq V\left(p_{c}(t)\right) \leq c_{0}-\frac{1}{2} \underline{m}\left(k(t)-k^{*}\right)^{2}+c_{1}\left(1+\lambda^{-1}\right)\left(k(t)-k^{0}\right)
$$

for all $t \in\left[t_{0}, \omega\right)$. Therefore, we see that the monotone increasing function $k(\cdot)$ is bounded. This, in turn, implies boundedness of $V\left(p_{c}(\cdot)\right)$ and so $p_{c}(\cdot)$ is bounded. It immediately follows that $q_{c}(\cdot)$ is bounded. By assumptions A4 and A5, we see that $\left(q_{r}, \dot{q}_{r}\right)(\cdot)$ is bounded. We have now shown that the solution $x(\cdot)$ is bounded and so $\omega=\infty$. Assertion (ii) of the theorem is now a consequence of boundedness and monotonicity of $k(\cdot)$.

To prove assertion (iii), we argue as follows. Observe that

$$
\left.d_{\lambda}\left(p_{c}(t)\right)\left\|q_{c}(t)\right\| \leq d_{\lambda}\left(p_{c}(t)\right) \lambda^{-1} \| p_{c}(t)\right)\left\|\mid q_{c}(t)\right\| \leq \lambda^{-1}\left\|q_{c}(t)\right\| \dot{k}(t)
$$

and so, by boundedness of $q_{c}(\cdot)$, there exists positive scalar $c_{2}$ such that

\section{$\eta^{2} d_{\lambda}\left(p_{c}(t)\right)\left\|q_{c}(t)\right\| \leq c_{2} \dot{k}(t)$}

for almost all $t \geq t_{0}$. Writing $c_{3}:=\underline{m}\left(k^{*}-k^{0}\right)+c_{2}$, we conclude that
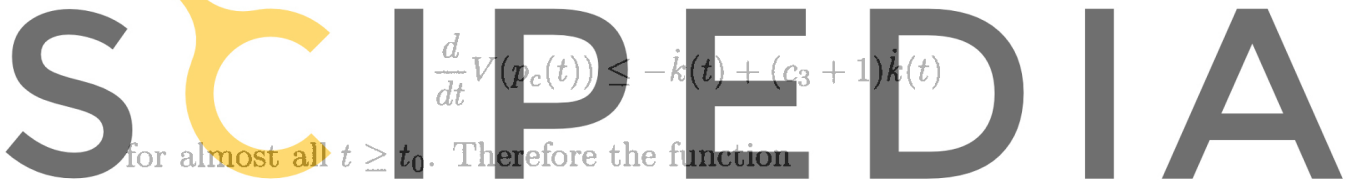

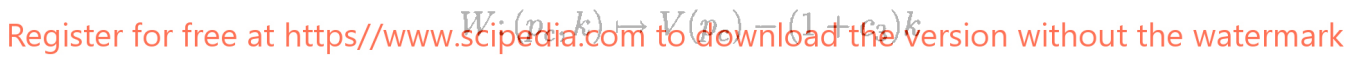

is such that

$$
\frac{d}{d t} W\left(p_{c}(t), k(t)\right) \leq-\dot{k}(t) \leq-d_{\lambda}\left(p_{c}(t)\right)\left\|p_{c}(t)\right\|
$$

for almost all $t \geq t_{0}$. Boundedness of the solution $x(\cdot)$ ensures that it has nonempty $\omega$-limit set $\Omega$. Since the solution approaches its $\omega$-limit set, we first prove that

$$
d_{\lambda}\left(p_{c}(t)\right) \rightarrow 0 \quad \text { as } t \rightarrow \infty
$$

by showing that $d_{\lambda}\left(\bar{p}_{c}\right)=0$ for all $\bar{x}=\left(\bar{q}_{r}, \bar{v}_{r}, \bar{q}_{c}, \bar{p}_{c}, \bar{k}\right) \in \Omega$. Suppose otherwise. Then there exists $\bar{x}=\left(\bar{q}_{r}, \bar{v}_{r}, \bar{q}_{c}, \bar{p}_{c}, \bar{k}\right) \in \Omega$ and $\epsilon>0$ such that $d_{\lambda}\left(\bar{p}_{c}\right)\left\|\bar{p}_{c}\right\|>2 \epsilon$. By continuity, there exists $\tilde{\delta}>0$ such that

$$
\left\|\xi-\bar{p}_{c}\right\|<\bar{\delta} \Longrightarrow d_{\lambda}(\xi)\|\xi\|>\epsilon .
$$

Since $\bar{x}$ is an $\omega$-limit point, there exists a sequence $\left(t_{j}\right)$ with $t_{j} \rightarrow \infty$ and

$$
x\left(t_{j}\right)=\left(q_{r}\left(t_{j}\right), \dot{q}_{r}\left(t_{j}\right), q_{c}\left(t_{j}\right), p_{c}\left(t_{j}\right), k\left(t_{j}\right)\right) \rightarrow \bar{x}
$$


as $j \rightarrow \infty$. By Assumptions A1-A6, it is readily verified that there exist $\bar{\delta}>0$ and $R>0$ such that

$$
\|x-\bar{x}\|<\bar{\delta} \Longrightarrow\left\|F_{\lambda}(t, x)\right\|<R .
$$

We may assume $\bar{\delta}<\tilde{\delta}$. By continuity of $W$,

$$
W\left(p_{c}\left(t_{j}\right), k\left(t_{j}\right)\right)-W\left(\bar{p}_{c}, \bar{k}\right)<\frac{\epsilon \bar{\delta}}{4 R}
$$

for all $j$ sufficiently large. Let $j^{*}$ be such that

$$
\left\|x\left(t_{j}\right)-\bar{x}\right\|<\frac{1}{2} \bar{\delta} \quad \forall j>j^{*} .
$$

\section{By (7) and (9), it follows that}

$$
\forall t \in\left[t_{j}, t_{j}+(\bar{\delta} /(3 R))\right]
$$

which holds for all $j>j^{*}$. Therefore, using (8), we have for all $j>j^{*}$

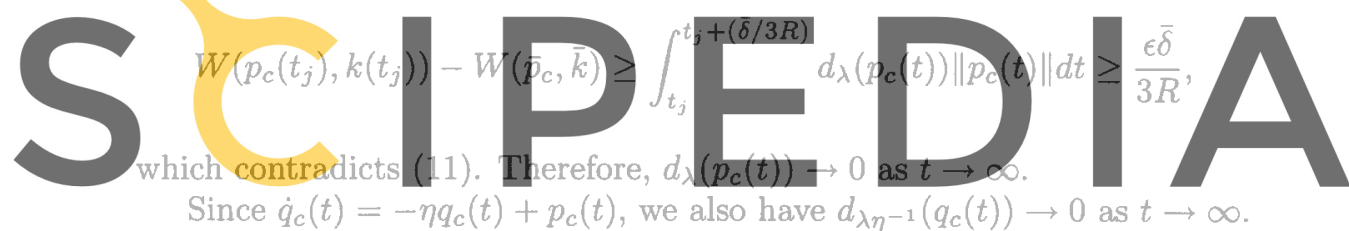

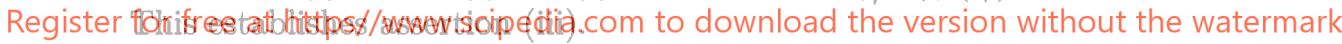

Finally, assertion (iv) is a direct consequence of Assumptions A4 and A5.

REMARKS. By the above theorem, we see that the proposed adaptive feedback strategy ensures a form of practical stability for the system class $\Sigma$. In essence, for any prescribed $\lambda>0$, the subsystem state $\left(q_{c}(t), p_{c}(t)\right)$ is asymptotic to that ball (centered at zero in $\mathbb{R}^{2 n_{c}}$ ) of radius $\lambda \sqrt{1+\eta^{-1}}$, the remaining subsystem state $\left(q_{r}(t), \dot{q}_{r}(t)\right)$ is asymptotic to a ball (centered at zero in $\mathbb{R}^{2 n_{r}}$ ) of radius $c \lambda$; however, the scale factor $c>0$ depends on the unknown function $h$ and so is not computable from a priori system information.

\section{ILLUSTRATIVE EXAMPLE}

Consider a 10-story base isolated shear building as shown in Figure 1 and described by (2).The masses of the base and of each floor of the 


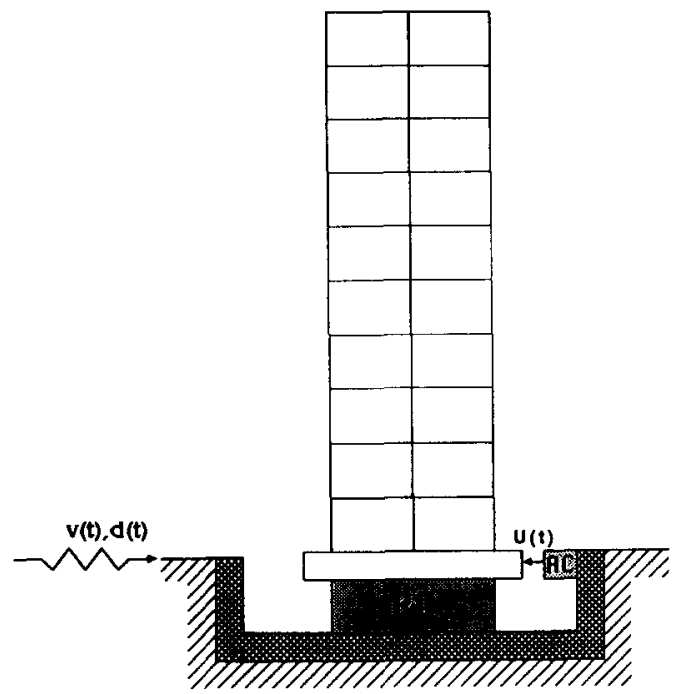

FIG. 1. Building structure with a hybrid control system. PC: base isolator; AC active controller.

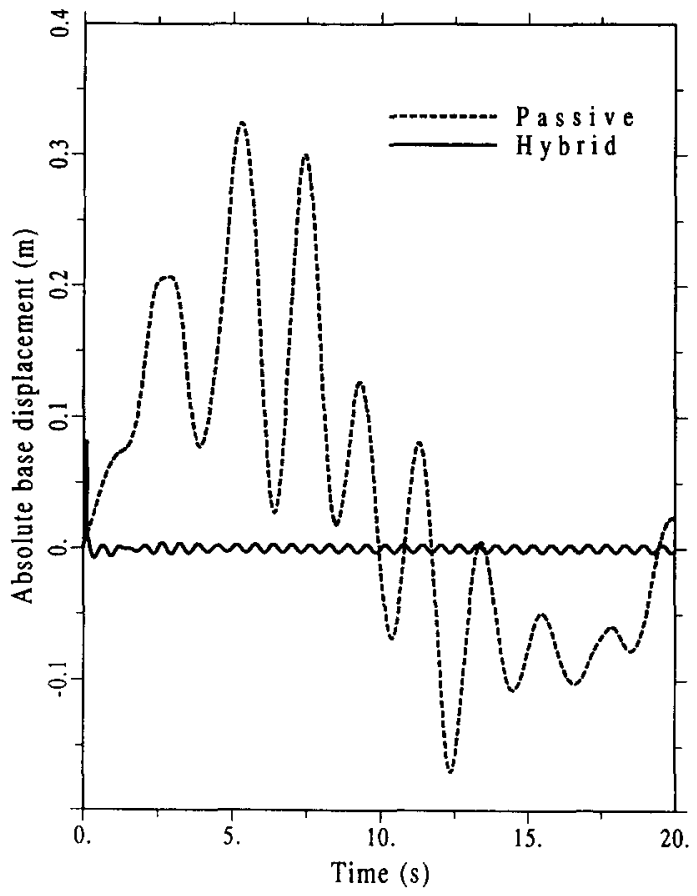

FIG. 2. Absolute base displacement response for passive and hybrid cases. 


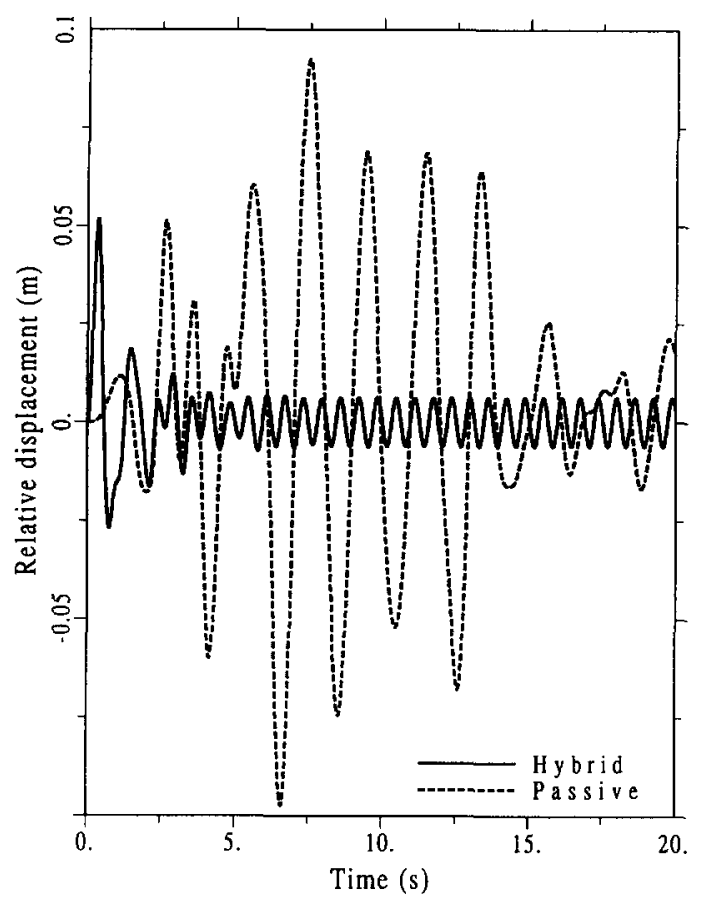

FIG. 3. Displacement of the 10th floor relative to the base for passive and hybrid cases.

building are $6 \times 10^{5} \mathrm{Kg}$. The stiffness of the base is $k_{b}=7 \times 10^{8} \mathrm{~N} / \mathrm{m}$ and its damping ratio is 0.1 . The stiffness of the building varies in $5 \times 10^{7} \mathrm{~N} / \mathrm{m}$ between floors, from $9 \times 10^{8} \mathrm{~N} / \mathrm{m}$ the first one to $4.5 \times 10^{8} \mathrm{~N} / \mathrm{m}$ the top one, while the damping ratio is 0.05 . The nonlinear force $f$ produced by the base isolation device on the base has elastoplastic hysteretic characteristics. The purpose of this example is to show the effectiveness of the control law (5) and (6) when applied to the above described structural system. To do this, the first step is to identify the function $\gamma_{\mu}$ appearing in (6). In this case, $\mu\left(q_{c}\right)=1$. To obtain the function $\gamma$, according to Assumption A6, we first need to identify function $g_{c}$ for the case of equations (2). Comparing subsystems $\Sigma_{c}$ in (1) and (2), it is observed that

$$
\begin{aligned}
g_{c}\left(t, q_{r}(t), \dot{q}_{r}(t), q_{c}(t), \dot{q}_{c}(t)\right)= & {\left[c_{b}+J^{T} C J\right] \dot{q}_{c}+\left[k_{b}+J^{T} K J\right] q_{c} } \\
& -J^{T} C \dot{q}_{r}-J^{T} K q_{r}-c_{b} v-k_{b} d \\
& +f\left(q_{c}, \dot{q}_{c}, d, v\right) .
\end{aligned}
$$




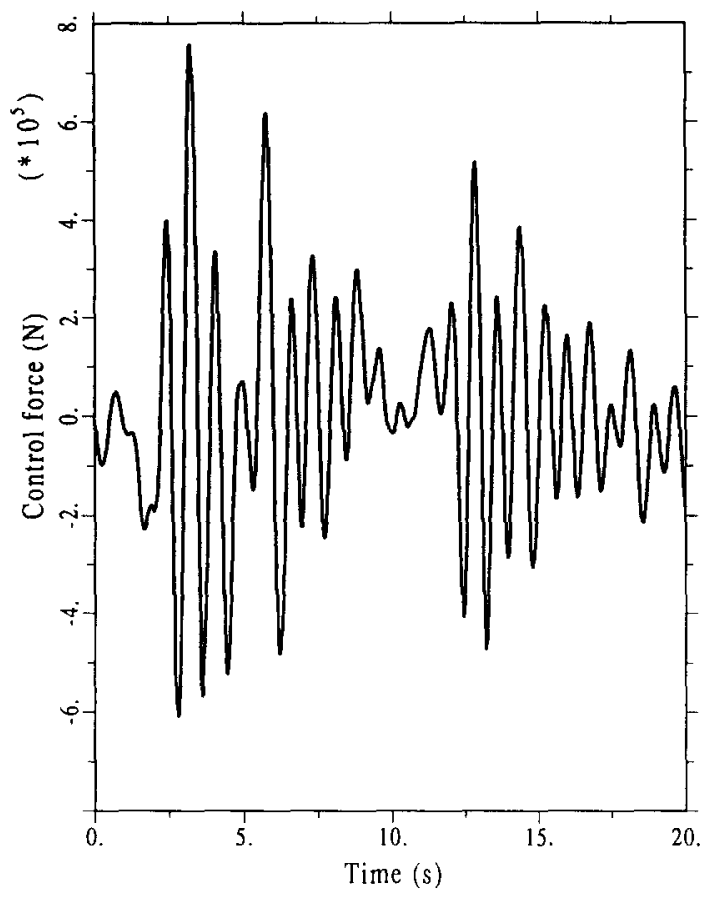

FIG. 4. Active control force.

According to the elastoplastic behavior considered for the isolator, the nonlinear force $f$ remains always bounded since it is limited by the yielding force. Thus, condition (4) reduces to

$$
\left\|f\left(q_{c}, \dot{q}_{c}, d, v\right)\right\| \leq \xi
$$

$\xi$ being an unknown scalar. Using now inequalities (3) and (13) in (12), it can be readily written

$$
\left\|g_{c}\left(t, q_{r}, \dot{q}_{r}, q_{c}, \dot{q}_{c}\right)\right\| \leq \epsilon \gamma\left(q_{r}, \dot{q}_{r}, q_{c}, \dot{q}_{c}\right)
$$

where $\epsilon$ is an unknown scalar and

$$
\gamma\left(q_{r}, \dot{q}_{r}, q_{c}, \dot{q}_{c}\right)=\left[q_{c}^{2}+\dot{q}_{c}^{2}+q_{r_{1}}^{2}+\cdots+q_{r_{n}}^{2}+\dot{q}_{r_{1}}^{2}+\cdots+\dot{q}_{r_{n}}^{2}+1\right]^{1 / 2}
$$

Using this function, the control law (5) and (6) is now applied to compute the active control $u$ for all time $t$. 


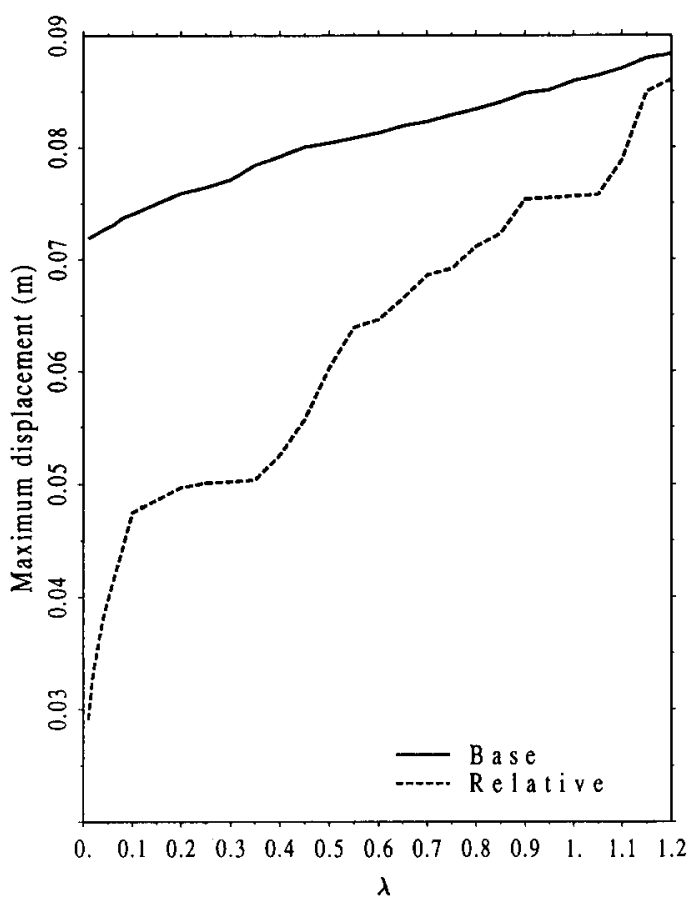

FIG. 5. Variation with $\lambda$ of the maximum absolute base displacement and maximum displacement of the 10th floor relative to the base.

The application of the control law has been numerically simulated and some of the results are included in this section. In all the tests, parameter $\eta$ has been chosen equal to 1 . Figures 2 and 3 show the time histories of the absolute displacement and the displacement of the 10th floor of the structure relative to the base. The seismic excitation has been that of the El Centro (1940) earthquake. In both figures the responses for the passive and hybrid cases are compared. It can be observed that for the hybrid case the displacement response rapidly enters within a bounded region around zero. This shows a behavior as expected from the stability analysis performed in previous Section 3.1. The corresponding control force, plotted in Figure 4, remains within an acceptable range. $\lambda$ is the most significant parameter in the implementation of this control law since it defines the size of the stability region. In order to assess the influence of this parameter in the effectiveness of the control law, Figure 5 displays the maximum values of the absolute base displacement and the displacement of the 10th floor relative to the base as 


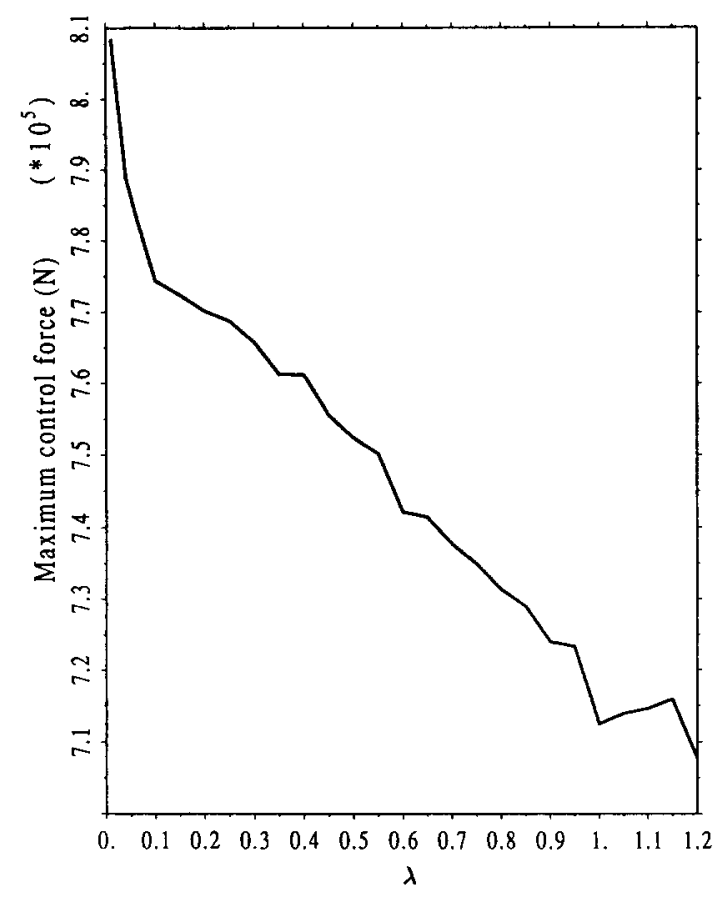

FIG. 6. Variation with $\lambda$ of the maximum active control force.

a function of $\lambda$. It can be observed that the smaller the value of $\lambda$ is, the smaller the controlled displacements that are obtained since it implies a more demanding control objective. This behavior is also apparent in Figure 6 , where the maximum value of the control force is plotted against $\lambda$.

Financial support from CIRIT (Government of Catalonia) and the British Council/Spanish Ministry of Education (Acciones Integradas Programme) is greatly appreciated.

\section{REFERENCES}

1 G. Leitmann, Deterministic control of uncertain systems via a constructive use of Lyapunov stability theory, in Proc. of the 14th IFIP Conference on System Modelling and Optimization, Lecture Notes in Control and Information Sciences, Vol. 143, (H. J. Sebastian and K. Tammer, Eds.), Springer-Verlag, New York, 1990, pp. 38-55. 
2 J. M. Kelly, G. Leitmann, and A. Soldatos, Robust control of base-isolated structures under earthquake excitation, J. Optimization Theory Appl. 53:159181 (1987).

3 Y. H. Chen and J. Piontek, Robust modal control of distributed parameter systems with uncertainty, in Proc. American Control Conference, San Diego, CA, 1990, pp. 2014-2019.

4 J. Rodellar, G. Leitmann, and E. P. Ryan, Output feedback control of uncertain coupled systems, Internat. J. Control 58(2):445-457 (1993).

5 M. Corless and G. Leitmann, Adaptive control of systems containing uncertain functions and unknown functions with uncertain bounds, J. Optim. Theory Appl. 41:155-168 (1983).

6 E. P. Ryan, A universal adaptive stabilizer for a class of nonlinear systems, Systems Control Lett. 16:219--218 (1991).

7 R. I. Skinner, W. H. Robinson, and G. H. McVerry, An Introduction to Seismic Isolation, Wiley, Chichester, 1993.

8 J. Inaudi, F. López Almansa, J. M. Kelly, and J. Rodellar, Predictive control of base isolated structures, Earthquake Eng. Struct. Dynam. 21:471-482 (1992).

9 J. Inaudi and J. M. Kelly, A simple active isolation scheme, in 8th VPI and SU Symposium on Dynamics and Control of Large Structures, Blacksburg, VA, 1991, pp. 219-231.

10 J. N. Yang, Z. Li, A. Danielians, and S. C. Liu, Aseismic hybrid control of nonlinear and hysteretic structures, I and II, J. Eng. Mech. 118(7):1423-1456 (1992).

11 S. Nagarajaiah, M. Riley, A. Reinhorn, and M. Shinozuka, Hybrid control of sliding isolated bridges, in Proc. 1992 Presure Vessels and Piping Conf., ASME/PVP-237, 2:83-89 (1992).

12 M. Q. Feng, M. Shinozuka, and S. Fujii, Friction-controllable sliding isolated system, J. Eng. Mech. 119(9):1845-1864 (1993). 